\title{
Understanding MRI: basic MR physics for physicians
}

\author{
Stuart Currie, ${ }^{1}$ Nigel Hoggard, ${ }^{1}$ lan J Craven, ${ }^{1}$ Marios Hadjivassiliou, ${ }^{2}$ \\ lain D Wilkinson ${ }^{1}$
}

${ }^{1}$ Academic Unit of Radiology, University of Sheffield, Royal Hallamshire Hospital, Sheffield, UK

${ }^{2}$ Department of Neurology, Royal Hallamshire Hospital Sheffield, UK

\section{Correspondence to} Dr Stuart Currie, Academic Unit of Radiology, University of Sheffield, Floor C, Royal Hallamshire Hospital, Glossop Road, Sheffield S10 2JF, UK:

s.currie@sheffield.ac.uk

Received 24 July 2012 Revised 9 August 2012 Accepted 14 November 2012 Published Online First 7 December 2012

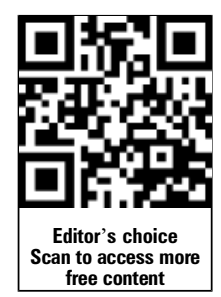

To cite: Currie $S$, Hoggard N, Craven IJ, et al. Postgrad Med J

2013;89:209-223.

\section{ABSTRACT}

More frequently hospital clinicians are reviewing images from MR studies of their patients before seeking formal radiological opinion. This practice is driven by a multitude of factors, including an increased demand placed on hospital services, the wide availability of the picture archiving and communication system, time pressures for patient treatment (eg, in the management of acute stroke) and an inherent desire for the clinician to learn. Knowledge of the basic physical principles behind MRI is essential for correct image interpretation. This article, written for the general hospital physician, describes the basic physics of MRI taking into account the machinery, contrast weighting, spin- and gradientecho techniques and pertinent safety issues. Examples provided are primarily referenced to neuroradiology reflecting the subspecialty for which MR currently has the greatest clinical application.

\section{INTRODUCTION}

For years, access to images from MR studies was limited to the reporting radiologist with clinicians seeing them briefly at multidisciplinary team meetings. That has now changed, largely due to the introduction of the picture archiving and communication system. More frequently, clinicians review MRI before seeking specialist radiological opinion. However, knowledge of the basic physical principles underlying MRI acquisition is fundamental to image interpretation.

This article, written for the general hospital physician, describes the basic physics of MRI taking into account the machinery, contrast weighting, spin- and gradient-echo techniques and pertinent safety issues. Examples provided are primarily referenced to neuroradiology reflecting the subspecialty for which MR currently has the greatest clinical application.

\section{THE EQUIPMENT}

The MR system comprises two main groups of equipment. The first is the control centre, which is positioned where the operator sits. The control centre houses the 'host' computer with its graphical user interface. Its associated electronics and power amplifiers are usually situated in an adjacent room and connect to the second equipment group. This second group of equipment is housed within the machine in which the patient lies. It contains the parts of the MR system that generate and receive the MR signal and include a set of main magnet coils, three gradient coils, shim coils and an integral radiofrequency (RF) transmitter coil ${ }^{1}$ (figure 1). Due to the necessary use of RF electromagnetic waves or radio waves (see below), the room that contains this second set of equipment needs to keep potential sources of electromagnetic noise out and its own RF in. This is achieved by enclosing the magnet and its associated coils within a special, copper-lined examination room, forming what the Physics community calls a Faraday shield.

\section{Magnets}

Recall the principles of the Maxwell equations that indicate that when an electric current flows through a wire, a magnetic field is induced around the wire. Resistance to the flow of the electric current can be reduced to negligible levels if a special metal conductor is cooled substantially. In this situation, lower resistance allows the use of high electric currents to produce high-strength magnetic fields, with little heat disposition. This principle is employed in the generation of superconducting magnets: the type of magnet typically used in clinical MR systems. The main magnet coils, made of a superconducting metal-alloy, are cooled (close to absolute zero, $\sim 4^{\circ} \mathrm{K}$ or $-269^{\circ} \mathrm{C}$ ) using expensive cryogenic liquid helium. ${ }^{2}$

The main magnet coils generate a strong, constant magnetic field $\left(\mathrm{B}_{0}\right)$ to which the patient is exposed. The strength of the magnetic field is measured in units of Tesla, $(\mathrm{T})$. One Tesla is equivalent to approximately 20000 times the earth's magnetic field. Currently, most clinical MR systems are superconducting and operate at $1.5 \mathrm{~T}$ or $3 \mathrm{~T}$. Field strengths reaching 9.4 $\mathrm{T}$ have been used in human imaging, albeit typically in research.

\section{Gradient coils}

The MR system uses a set coordinates to define the direction of the magnetic field. Gradient coils representing the three orthogonal directions $(x, y$ and $\mathrm{z}$ ) lie concentric to each other within the main magnet (figure 1). They are not supercooled and operate relatively close to room temperature. Each gradient coil is capable of generating a magnetic field in the same direction as $\mathrm{B}_{0}$, but with a strength that changes with position along the $\mathrm{x}, \mathrm{y}$ or $\mathrm{z}$ directions, depending on which gradient coil is used. The magnetic field generated by the gradient coils is superimposed on top of $B_{0}$ so that the main magnetic field strength varies along the direction of the applied gradient field (figure 2).

\section{RF coils}

RF coils, so named because the frequency of electromagnetic energy generated by them lies within the megahertz range, are mounted inside the gradient coils and lie concentric to them and to each other. They may be thought of as the 'antenna' of the MRI system and accordingly they have two main purposes: to transmit RF energy to the tissue 
Figure 1 Schematic demonstrating the relative positions of the different magnet coils comprising the MR machine. The patient is positioned within the bore of the machine and is surrounded by coils that lie concentric to each other and in the following order (from furthest to closest to the patient): main magnet coils, gradient coils and radiofrequency (RF) coils. For neuroimaging, a further RF coil is placed around the patient's head to improve signal to noise ratio.

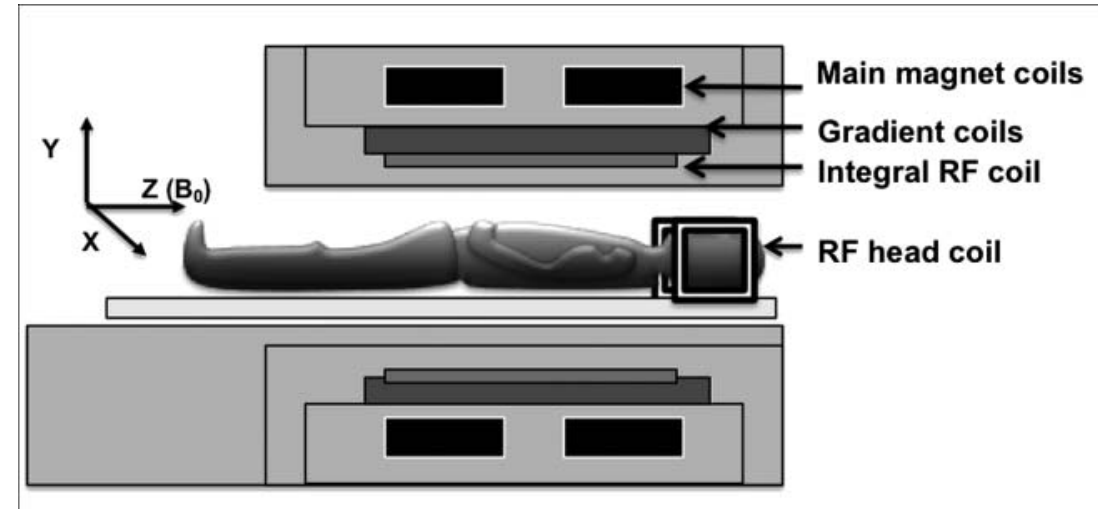

of interest and to receive the induced RF signal back from the tissue of interest. ${ }^{2}$

Some RF coils perform the dual role of transmission and reception of RF energy whereas others transmit or receive only. For neuroimaging, a separate RF receiver coil that is tailored to maximise the signal from the brain is usually applied around the patient's head to detect the emitted MR signals.

The RF field is also referred to as the $B_{1}$ field. When switched on, the $\mathrm{B}_{1}$ field combines with $\mathrm{B}_{0}$ to generate $\mathrm{MR}$ signals that are spatially localised and encoded by the gradient magnetic fields to create an MRI. ${ }^{1}$ The output signal picked up by the receive coil is digitised and then sent to a reconstruction computer processor to yield the image after complex mathematical manipulation. ${ }^{3}$

\section{Shim coils}

Localisation of the MR signal requires good homogeneity within the local magnetic field. In other words, the more uniform the magnetic field the better. However, placement of an object (including a patient) within the main $\mathrm{B}_{0}$ field creates local susceptibility effects and reduces homogeneity. Shimming refers to adjustments made to the magnet to improve its

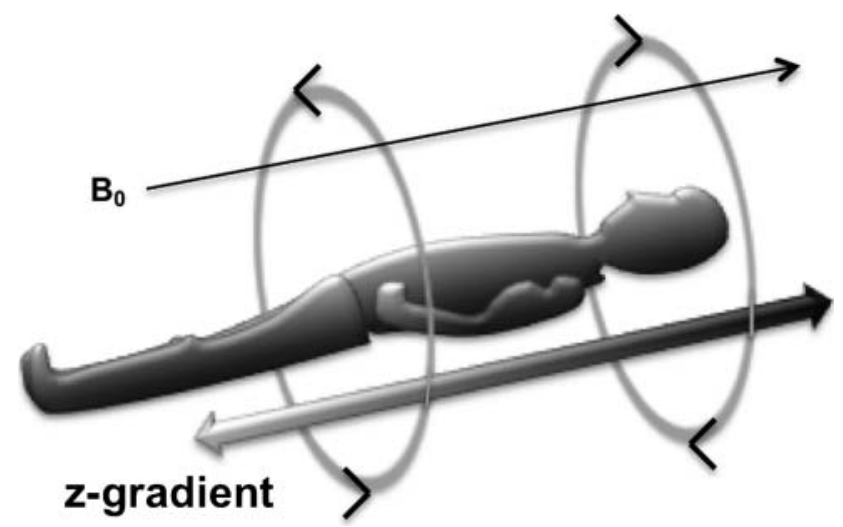

Figure 2 Image shown depicts the generation of a gradient in $\mathrm{B}_{0}$ in the $\mathbf{Z}$ direction. For a standard clinical MR system, this is accomplished using two coils in which the current flowing through them runs in opposite directions to each other (the so-called Maxwell pair type). The magnetic field at the centre of one coil adds to the $B_{0}$ field while the magnetic field at the centre of the other subtracts from $B_{0}$, thus creating a gradient in the $B_{0}$ field. ${ }^{29} X$ and $Y$ gradient coils (not shown) generally have a figure- 8 configuration. (These designs have started to be superseded by 'thumb-print' designs, which produce the desired gradients over the patient, but minimise any interfering field interacting with the main magnet's coils and containers.) homogeneity. Shimming can be passive or active. Passive shimming is achieved during magnet installation by placing sheets or little coins of metal at certain locations at the edge of the magnet bore (close to where the RF and gradient coils lie). Active shimming provides additional field correction around an object of interest through the use of shim coils, which are activated by electric currents controlled by the host computer, under the guidance of the scanner application software and the operator. Homogeneity of and hence variation in $\mathrm{B}_{0}$ is quoted in parts per million (ppm), that is, a fraction, of the static magnetic field over a specified spherical volume. For a $1 \mathrm{~T}$ system with a homogeneity of $1 \mathrm{ppm}$ over $40 \mathrm{~cm}$, no two points within $20 \mathrm{~cm}$ of isocenter differ by more than $0.000001 \mathrm{~T}^{4}$

\section{OBTAINING MR SIGNALS}

Origin of the MR signal: protons and 'little bar magnets'

The primary origin of the MR signal used to generate almost all clinical images comes from hydrogen nuclei. Hydrogen nuclei consist of a single proton that carries a positive electrical charge. The proton is constantly spinning and so the positive charge spins around with it. Recall that a moving electrical charge is called a current and that an electrical current generates a magnetic field. Thus, protons have their own magnetic fields and behave like little bar magnets (figure 3).

The magnetic field for each proton is known as a magnetic moment. Magnetic moments are normally randomly orientated. However, when an external magnetic field $\left(B_{0}\right)$ is applied they align either with (parallel) or against (antiparallel) the external field. The preferred state of alignment is the one that requires the least energy: that is, parallel to $\mathrm{B}_{0}$. Accordingly more protons align with $\mathrm{B}_{0}$ than against it. The difference in the number of protons aligning parallel and antiparallel to $\mathrm{B}_{0}$ is
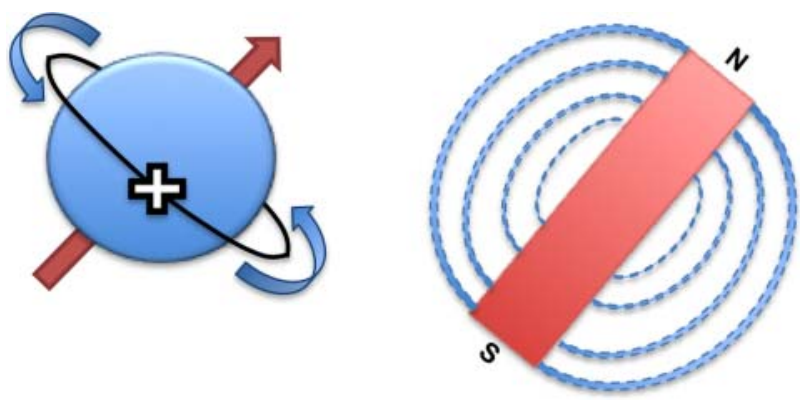

Figure 3 Protons possess a positive charge and are constantly spinning around their own axes. This generates a magnetic field making protons similar to bar magnets. This figure is only reproduced in colour in the online version. 
typically very small but ultimately depends on the strength of $\mathrm{B}_{0}$ as well as the temperature of the sample. As a rough estimate, for about 10 million protons aligning parallel to $\mathrm{B}_{0}$, there are approximately 10000007 aligning antiparallel to the external magnetic field. ${ }^{5}$

\section{Precession}

When put in an external static magnetic field, the overall effect on a group of protons (that individually are aligned either parallel or antiparallel to $\mathrm{B}_{0}$ ) means that the group of spins classically move in a particular way called precession. Precession can be likened to the movement of a spinning top. When spun, the top wobbles but does not fall over and the axes of the top circles form a cone shape (figure 4).

The speed of precession, that is, how many times the protons precess per second, is measured as the precession frequency (also named the Larmor frequency, $\omega_{0}$, in $\mathrm{MHz}$ ) and determined by the Larmor equation:

$$
\omega_{0}=\gamma \mathrm{B}_{0}
$$

$\gamma$ Is a constant for a particular nuclear species (eg hydrogen) termed the gyromagnetic ratio. Its value for the proton is $42.6 \mathrm{MHz} / \mathrm{T}$. The Larmor equation indicates that precession frequency is proportional to the strength of the magnetic field.

\section{Longitudinal magnetisation}

Protons precessing parallel to $\mathrm{B}_{0}$ begin to cancel each other out in all directions bar one: the direction of the $\mathrm{z}$-axis, along $\mathrm{B}_{0}$ (figure 5A). The result is a sum magnetic field or sum magnetisation, often given the symbol $\mathrm{M}$, with the value $\mathrm{M}_{0}$. It is characteristically shown as a vector. As this sum magnetisation parallels the external magnetic field it is also referred to as longitudinal magnetisation (figure $5 \mathrm{~B}$ ).

The patient essentially becomes a magnet with a magnetic vector aligned with $B_{0}$. The magnetic force of the patient (as it stands) cannot be measured as it is in the same direction as the external field. What is required is a magnetisation that lies at an angle to $\mathrm{B}_{0}$.

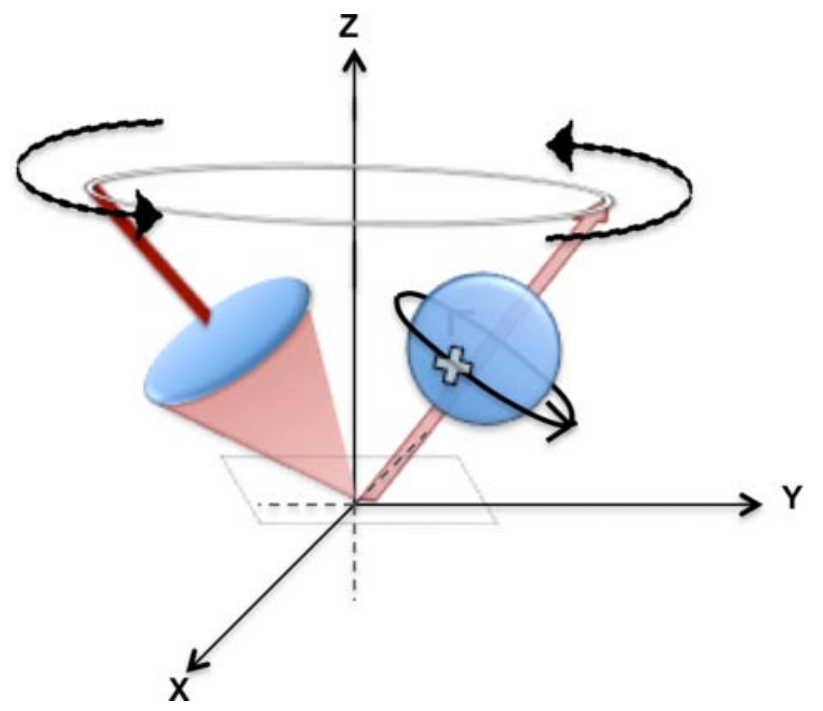

Figure 4 When exposed to an external magnetic field, protons precess. The movement of precession can be likened to the wobbling motion seen when a spinning top is spun. The handle of the spinning top follows a circular path. This figure is only reproduced in colour in the online version.

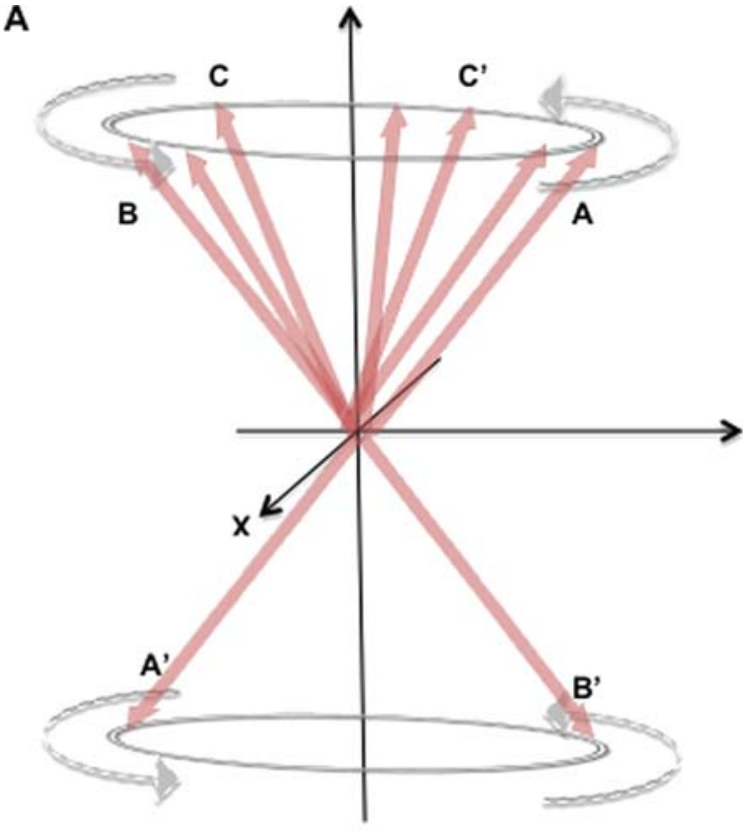

B

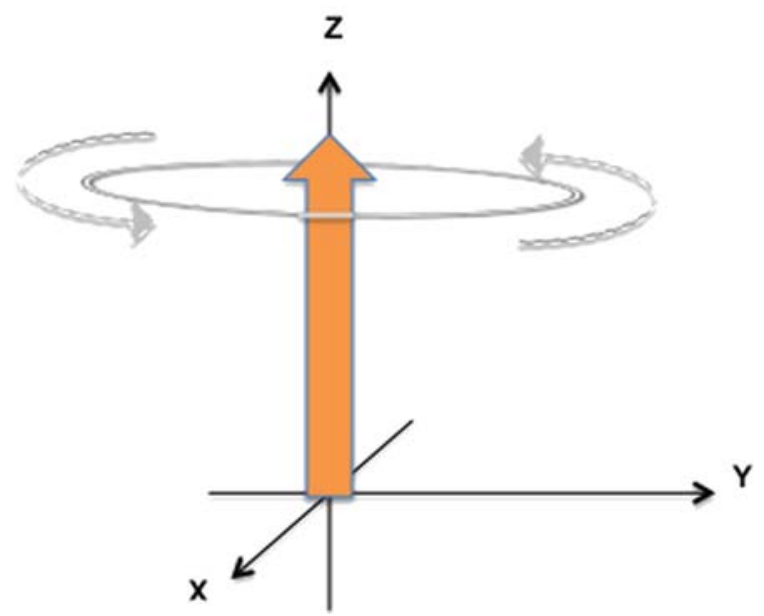

Figure 5 Longitudinal magnetisation. For simplicity protons are now shown as vectors. (A) The magnetic moments of protons precessing in the external magnetic field begin to cancel each other out. Opposing protons $A$ and $A^{\prime}$ and $B$ and $B^{\prime}$ 'neutralise' each other leaving a net number of protons lying parallel to $B_{0}$. Protons precessing parallel to $B_{0}$ also begin to cancel each other out. C (pointing to the left) and $C^{\prime}$ (pointing to the right) oppose and neutralise each other. This occurs in all directions bar one: the direction of the $z$-axis, along $B_{0}$. The result is a sum magnetic field that is typically depicted as a vector (B). This figure is only reproduced in colour in the online version.

\section{RF pulses and transverse magnetisation}

With the patient in the magnet and possessing longitudinal magnetisation, RF pulses are switched on and off. The purpose of the RF pulse is to disturb the protons so that they fall out of alignment with $\mathrm{B}_{0}$. This disturbance occurs through the transference of energy from the RF pulse to the protons. This can only occur when the RF pulse has the same frequency as the precessional frequency of the protons, a phenomenon called resonance; hence the term magnetic resonance imaging. ${ }^{5}$ Accordingly, RF pulses are set at the Larmor frequency.

The activation of an RF pulse has two main effects on the protons. First, some protons gain energy and move to the 
higher energy state of being antiparallel to $\mathrm{B}_{0}$. Consequently, opposing 'little bar magnets' (those parallel and antiparallel to $\mathrm{B}_{0}$ ) once again cancel each other out, resulting in a reduction in overall longitudinal magnetisation. Second, the RF pulse causes the protons to move in phase (ie, in the same direction, at the same time) with each other rather than in random directions. The result is transverse magnetisation in which a new magnetisation vector is created in the $\mathrm{x}-\mathrm{y}$ plane and moves in line with the precessing protons at the Larmor frequency.

The transverse magnetisation vector is a moving magnetic field (rotating at the Larmor frequency) and, as such, if a conductive receiver coil is placed in proximity, an alternating voltage will be induced across it. This in turn generates an electrical current, which can be picked up (like an antenna would pick up radio waves) forming an MR signal. As soon as the RF pulse is switched off the protons start to fall out of phase with each other and also return to a lower energy state, that is, the protons relax. Relaxation occurs in two different ways. Transverse magnetisation begins to disappear, a process called transverse (or T2 ('Time' 2)) relaxation and the longitudinal magnetisation starts to return to its original value, a process termed longitudinal (or T1 ('Time' 1)) relaxation (figure 6).

\section{$\mathrm{T} 1$ relaxation and $\mathrm{T} 1$ values}

T1 relaxation is the process whereby protons exchange energy with their surroundings to return to their lower energy state and in doing cause the restoration of longitudinal magnetisation. The rate at which this occurs is dependent on the tumbling rate of the molecule in which the proton resides. Tumbling rate describes the rate of molecular motion. As molecules tumble they generate a fluctuating magnetic field to which protons in neighbouring molecules are subjected. Energy exchange (and therefore T1 relaxation) is more favourable when this fluctuating magnetic field is close to the Larmor frequency.

Different molecules have different tumbling rates and as a result they also differ in their efficiency at T1 relaxation. Free water (unbound/unrestricted) has a small molecular size and tumbles much too quickly to be effective at T1 relaxation. Similarly, hydrogen protons bound to large macromolecules (eg, membrane lipids) tumble very slowly and also demonstrate low
Figure 6 Recovery of longitudinal magnetisation following a $90^{\circ}$ radiofrequency (RF) pulse. (A) Protons aligned with $B_{0}$ produce a sum vector with longitudinal magnetisation. (B) When an RF pulse is switched on longitudinal magnetisation decreases and transverse magnetisation propagates. Alternatively, it can be said that the sum magnetisation 'tilts' to the side. An RF pulse that abolishes longitudinal magnetisation to zero while inducing transverse magnetisation is called a $90^{\circ}$ (saturation) pulse as the sum magnetisation vector is seen to tilt or flip $90^{\circ}$. Immediately following an RF pulse, protons precess in phase in the transverse plane, depicted by a single vector (arrow) in the lower circle. (C) After the $90^{\circ} \mathrm{RF}$ pulse, protons fall out of phase (now multiple vectors in the lower circle), transverse magnetisation decreases and longitudinal magnetisation begins to recover. During this process, the whole system continues precessing and so the sum vector takes a spiralling motion (D). Recovery of longitudinal magnetisation is termed $\mathrm{T} 1$ relaxation and loss of transverse magnetisation is called $\mathrm{T} 2$ relaxation. This figure is only reproduced in colour in the online version.
A
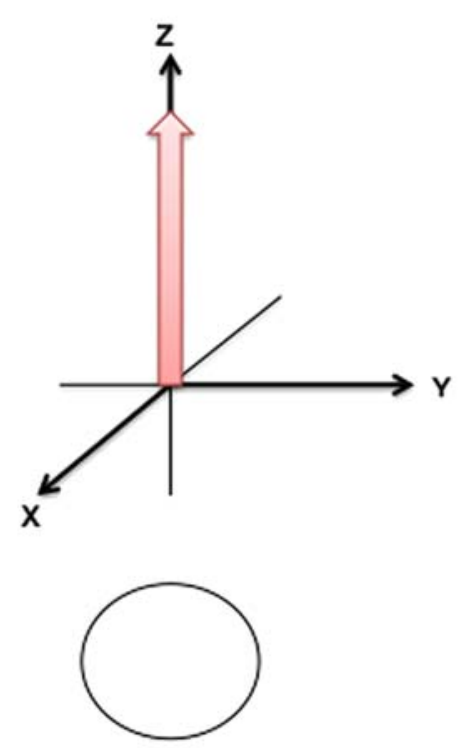

C
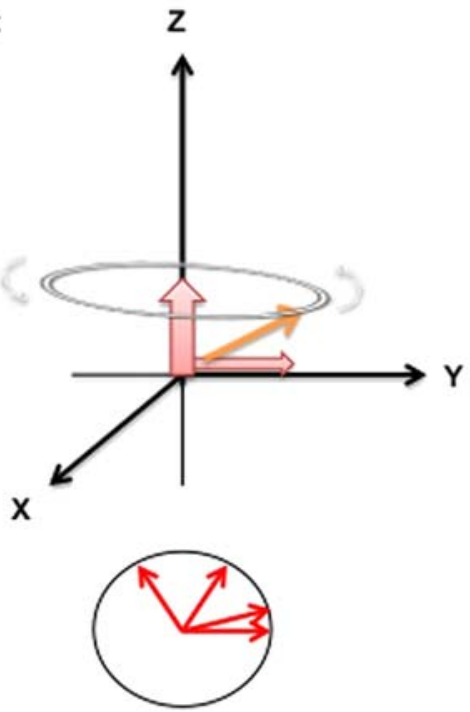

B

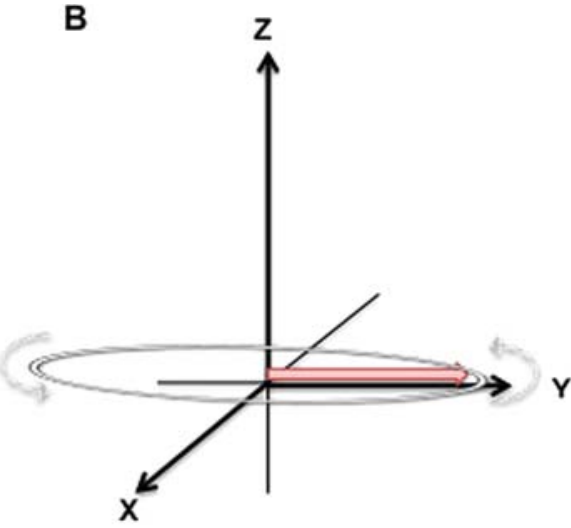

D $\quad$ z

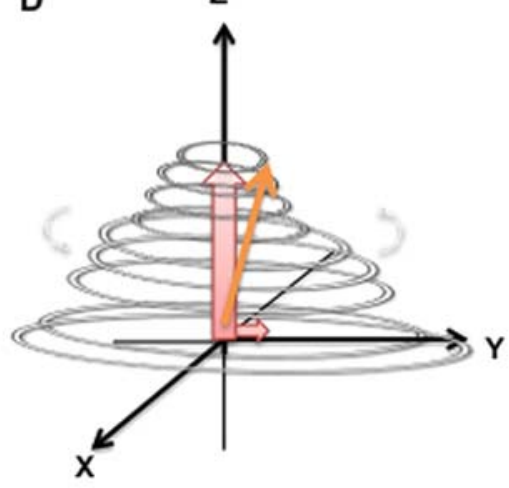

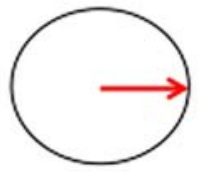

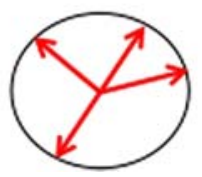


efficiency at T1 relaxation. Accordingly, both free water (4000 msec) and bound hydrogen have relatively long T1 relaxation times. Conversely, when water is partially bound (to proteins for example) its tumbling rate can be slowed to a rate more in line with the Larmor frequency. As a result, the T1 value of bound or structured water is much less than free water ( 400-800 msec). ${ }^{4}$ Fat typically has a short T1 value. This is because the carbon bonds at the ends of the fatty acids have frequencies near the Larmor frequency, allowing effective energy transfer. ${ }^{5}$

As T1 relaxation requires an exchange of energy between protons and their surroundings it is also termed spin-lattice relaxation. The term lattice is a throwback to early nuclear MR studies in solids, in which the external environment was literally a crystalline lattice of molecules. ${ }^{4}$

As not all protons return to their original energy state at the same time, T1 relaxation is more of a continuous process. Plotting the recovery of longitudinal magnetisation over time (after the RF pulse is switched off) produces an exponential curve, called the T1 curve. It is difficult to exactly pinpoint the end of longitudinal relaxation and so T1 (and similarly for T2) is not defined as the time of completion of longitudinal relaxation. Rather, T1 is a time constant that is used to describe how fast the process of T1 relaxation takes. Specifically, T1 is the time taken for longitudinal magnetisation to regrow from 0 to $\left(1-\mathrm{e}^{-1}\right)$, or approximately $63 \%$ of its final value. $1 / \mathrm{T} 1$ defines the longitudinal relaxation $\operatorname{rate}^{7}$ (figure $7 \mathrm{a}$ ).

\section{$\mathrm{T} 2$ and $\mathrm{T}^{*}$ relaxation}

Transverse relaxation describes the process whereby protons fall out of phase in the $x-y$ plane and transverse magnetisation decreases and disappears. There are two causes for this loss of phase coherence. The first, T2 relaxation results from slowly fluctuating magnetic field variations (inhomogeneity) within the local tissue. That is, the magnetic spin of protons is influenced by small magnetic fields from neighbouring nuclei. This results in the random fluctuation of the Larmor frequency of individual protons causing an exchange of energy between proton spins, which leads to loss of phase coherence across a population of protons. ${ }^{8}$ The internal inhomogeneity of spins (protons) influencing other neighbouring spins have lead to the term spin-spin relaxation also being used for $\mathrm{T} 2$ relaxation.

The second cause of loss of phase coherence is due to inhomogeneity within $\mathrm{B}_{0}$. Magnetic field variations result in slightly different Larmor frequencies for protons at different locations within the field. Unlike the random process of T2 relaxation, this de-phasing is caused by a constant and is potentially reversible. $\mathrm{T} 2 *$ (T2 star) relaxation is the name given to describe the effects that result from the combination of $\mathrm{T} 2$ relaxation and the de-phasing that results from inhomogeneity in $\mathrm{B}_{0}$. $\mathrm{T} 2 *$ relaxation determines the actual rate of decay observed when measuring a free induction decay (FID) signal (see later for details).

$\mathrm{T} 2$ is a constant describing the time taken for transverse magnetisation to decay to $\mathrm{e}^{-1}$, or about $37 \%$ of its initial value. Spin-spin interaction governs the speed of T2 relaxation and hence influences the T2 values for different tissues. Free water (eg, cerebrospinal fluid), comprising rapidly moving small molecules that are relatively far apart, will have less spin-spin interaction and therefore longer T2 values compared with water-based tissues that have a large macromolecular content (eg, grey matter), giving them more time to interact or 'chat' with each other.

In contrast to T1 relaxation, where energy transfer from the spin system must occur, T2 relaxation may proceed with or without overall energy loss. For human tissue transverse relaxation is typically a much faster process than longitudinal relaxation; hence, T2 values are always less than or equal to T1. For biological tissues, T1 is approximately 5-10 times longer than T2 (300-2000 msec vs 30-150 msec, respectively). ${ }^{4}$

\section{Free induction decay}

When an RF pulse is switched off, T1 and T2 relaxation occur simultaneously and independently. The protons continue to precess and the sum magnetisation vector follows a spiralling path whereby its direction and magnitude are constantly
Figure $7 \mathrm{~T} 1, \mathrm{~T} 2$ and $\mathrm{T} 2{ }^{*}$ relaxation. (A) T1 curve: Plotting the recovery of longitudinal magnetisation over time following the switching off of a radiofrequency (RF) pulse results in a T1 curve. (B) T2 curve: An $180^{\circ}$ refocusing pulse acts to 'combat' the effects of external magnetic field inhomogeneity by rephasing the protons. This results in a temporary gain in signal intensity at time echo time (TE) termed spin echo. A sequence of $180^{\circ}$ pulses results in a chain of spin echoes. Each subsequent echo will be of lower intensity due to T2 effects. A curve connecting the spin echo intensities is the T2 curve. (C) T2* curve: This curve results when $180^{\circ}$ refocusing pulses are not used. The signal decays much faster due to $\mathrm{T}^{*}$ effects. TR, repetition time. This figure is only reproduced in colour in the online version.

\section{Signal Intensity}

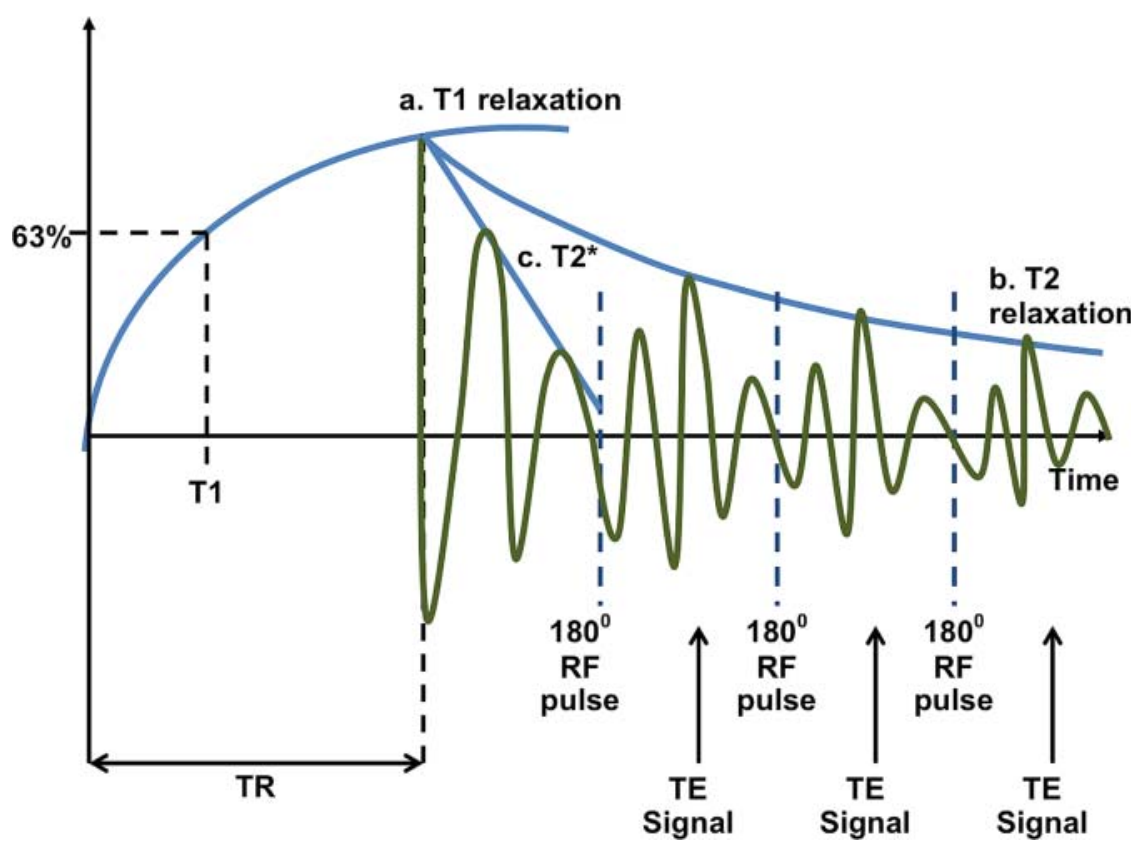


changing. Hence, an electrical signal is generated in a suitable receiver coil. The MR signal generated from the spiralling sum magnetisation vector is termed FID. It has its greatest magnitude immediately after the RF pulse is switched off and then decreases as both relaxation processes occur. It also has a constant frequency (resonant frequency) and consequently the FID signal takes the form of a sine wave with a rapidly decaying envelope (figure 8).

An FID is most commonly depicted with a $90^{\circ}$ pulse but a RF pulse of any flip angle can create an FID because some components of the longitudinal magnetisation are always tipped into the transverse plane. Theoretically, a $180^{\circ} \mathrm{RF}$ pulse should refrain from generating an FID. However, in practice, all $180^{\circ}$ pulses are imperfect and produce FID signals. ${ }^{4}$

FID is subjected to further disruption (de-phasing) by the magnetic field gradients that are used to localise and encode the MR signal. Consequently the signal generated by FID is not usually measured in MRI. Instead, it is common practice to generate and measure the MR signal in the form of an echo: typically a spin echo (SE) or a gradient echo (GRE). Echoes can be appreciated by considering how T1- and T2-weighted images are formed.

\section{T1-weighted images}

Contrast between tissues allows adjacent structures to be differentiated from one another. Contrast is determined by signal intensities, which in turn are governed (at least partly) by the T1 and T2 relaxation times of tissues within an image. An image in which the difference in signal intensity between tissues is predominantly due to differences in tissue $\mathrm{T} 1$ relaxation time is called a T1-weighted image. T1-weighted images are generated predominantly by manipulating the time between two RF excitation pulses, the so-called repetition time (TR) (figure 9).

The preceding example was simplified for ease of understanding. It should be appreciated that many parameters influence

\section{Signal Intensity}

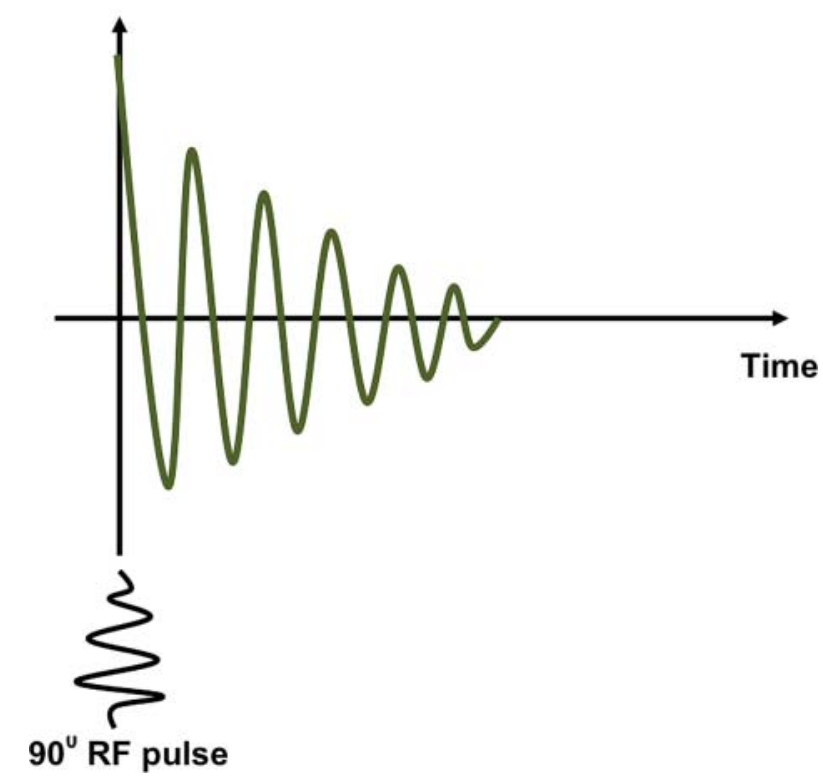

Figure 8 Free induction decay. Transverse magnetisation (with all protons rotating in phase) is at its greatest following an initial $90^{\circ}$ excitatory radiofrequency (RF) pulse. Its amplitude (along with signal intensity) then decreases as the protons begin to lose phase coherence. The resultant decay signal is termed free induction decay. This figure is only reproduced in colour in the online version. signal intensity and hence tissue contrast but in the example used T1 had the greatest influence. Contrast in images obtained at long TR will not be influenced by $\mathrm{T} 1$ but instead may be influenced by differences in the $\mathrm{T} 2$ or proton density of the tissues in question. Factors that influence MR signal intensity are listed in the box.

\section{T2-weighted images}

Recall that protons lose phase coherence following an RF pulse as a result of spin-spin interactions within the tissues (T2 relaxation) and because of inhomogeneity within the local static magnetic field (T2* relaxation). De-phasing caused by T2 relaxation is a random, irreversible process whereas the de-phasing caused by magnetic field inhomogeneity is potentially reversible. ${ }^{1}$ Application of an $180^{\circ} \mathrm{RF}$ pulse following an initial $90^{\circ} \mathrm{RF}$ pulse rotates the protons through $180^{\circ}$, effectively making the protons 'turn around' and precess, still in the $\mathrm{x}-\mathrm{y}$ plane, but in the opposite direction. Local field inhomogeneity remains and protons with a slightly faster Larmor frequency begin to catch up with slower protons. Eventually the protons come back into phase, which results in an increase in the amplitude of the MR signal. Maximum signal amplitude is reached at the echo time (TE). To achieve maximal signal, the $180^{\circ} \mathrm{RF}$ pulse must be applied at time $\mathrm{TE} / 2$. As the protons (spins) bounce back or echo following the application of the $180^{\circ} \mathrm{RF}$ refocusing pulse, the signal obtained is given the name SE (figure 10). Continued inhomogeneity in the static magnetic field means that protons will continue to lose phase coherence following an $180^{\circ} \mathrm{RF}$ refocusing pulse; however, it is possible to repeat the $180^{\circ} \mathrm{RF}$ refocusing pulse and obtain further SEs. Over time, the amplitude from each SE will decrease due to T2 effects. A curve connecting the SE intensities is called the T2 curve. T2* curve is the curve that results if $180^{\circ} \mathrm{RF}$ refocusing pulses are not used, that is, a curve that depicts the de-phasing caused by T2* effects (figure $7 \mathrm{~b}$ and $\mathrm{c}$ ).

Tissues have different T2 values. Brain for example has a shorter T2 than cerebrospinal fluid. TE is the time interval between the $90^{\circ} \mathrm{RF}$ pulse and the SE. It is one of the parameters whose value can be chosen by the operator of the MR machine in order to influence the signal intensities (hence contrast) between tissues. A much stronger signal is received when short TE (as opposed to long TE) is employed. However, at short TE (eg, <30 msec), differences in T2 have little influence on tissue contrast. Accordingly, T2-weighted images of the brain are obtained at long TE (eg, $80 \mathrm{msec}$ ) (figure 11).

A heavily T2-weighted image could be obtained at longer TE but loss of MR signal would impact on the signal to noise ratio making images potentially subdiagnostic.

\section{Factors influencing SE contrast and weighting}

Imaging parameters influencing the MR signal in the SE sequence include TE and TR. Values for both of these parameters are purposely chosen by the operator in order to influence the tissue weighting of the image. Short TR and short TE generates a T1-weighted image; short TR allows differences in longitudinal magnetisation to develop before the next $90^{\circ}$ excitation pulse while short TE limits the T2 effects. Long TR and long TE produces a predominantly $\mathrm{T} 2$-weighted image; long TR allows recovery of longitudinal magnetisation (thus limiting T1 effects) while at long TE T2 effects become pronounced. A different type of image is produced at long TR and short TE. Long TR and short TE limit T1 and T2 effects, respectively. When this occurs, the signal is predominantly influenced by the proton density of the tissues (figure $12 \mathrm{~A}-\mathrm{C}$ ). 
Figure 9 Repetition time (TR) and T1-weighting. Consider the following example in which two tissues, fat and fluid are being imaged where fat has shorter transverse and longitudinal relaxation times than fluid. An initial $90^{\circ}$ radiofrequency (RF) pulse is switched on and off, time passes (TR), a second $90^{\circ}$ RF pulse is switched on and off, MR signal is then sampled and an image is generated. As MR signal sampling occurs after the switching off of the second RF pulse, the signal intensity will be determined by the amount of transverse magnetisation at that point. This in turn will be governed by the value of longitudinal magnetisation of the two tissues immediately before the second $90^{\circ} \mathrm{RF}$ pulse is switched on. Choosing a long TR (eg, $1500 \mathrm{msec}$ ) would allow the tissues to recover their longitudinal magnetisation fully with no appreciable difference in the value of longitudinal magnetisation existing between the tissues. In this situation, the application of a second RF pulse with subsequent MR signal sampling would result in no discernible difference in MR signal between the tissues as the transverse magnetisation values for the tissues are essentially equal (A) (ie, no signal contrast). Choosing a short TR (20 msec) means that at the time of applying a second $90^{\circ}$ RF pulse tissues with a long T1 (fluid) will show less recovery in longitudinal magnetisation than tissues with a short T1 (fat). The tissues will possess different transverse magnetisation values (B) and, thus, will generate different signal intensities and allow greater contrast between tissues (C). This figure is only reproduced in colour in the online version.
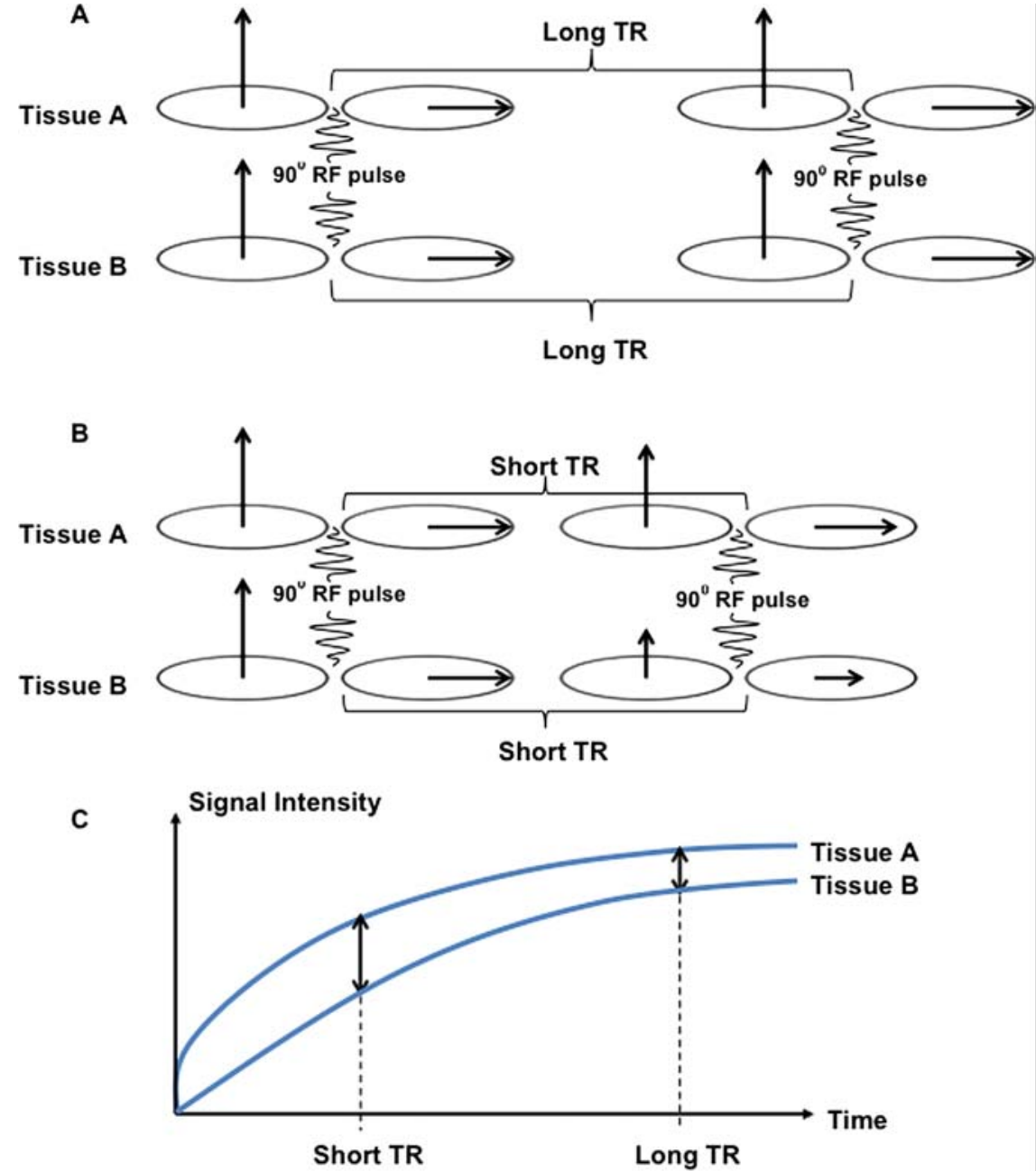

It should be appreciated that the choice of imaging parameters for all MRI sequences can influence the sensitivity of the test for the pathology in question. For example, the sensitivity for the detection of lesions in multiple sclerosis is dependent on TE (lesion visibility decreases with increasing TE). ${ }^{9}$

\section{Gradient echo}

SE sequences with their relatively long TR and TE are time consuming. This limits the number of patients who can be scanned

\section{Box 1 Factors that influence MR signal intensity}

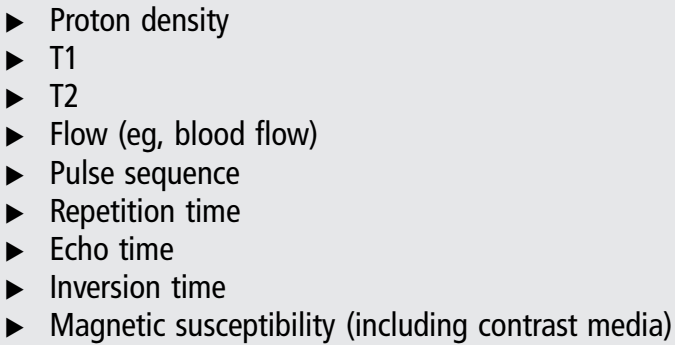

in a session and also risks movement artefact by a restless patient. Conversely, GRE sequences, which replace the $180^{\circ}$ refocusing RF pulse with magnetic field gradients, are relatively short. However, they do produce different contrasts. Magnetic field gradients produce a change in field strength and thus a change in Larmor frequency along a particular direction. Application of a gradient pulse after an initial RF pulse causes protons to rapidly de-phase along the direction of the gradient resulting in rapid decline in the FID signal. This loss of phase coherence can be reversed by applying a second magnetic field gradient with a slope of equal amplitude but in opposite direction to the first. As a result, protons move back into phase and return a signal called GRE. TE is the time taken between the beginning of FID (ie, generation of transverse magnetisation) following the initial RF pulse to the point at which the GRE reaches its maximum amplitude (figure 13 ).

\section{Differences between SE and GRE}

Several differences exist between the two techniques. First, TE can be shorter with GRE as only one RF pulse is used. Second, GRE sequences are typically used with low flip angle excitations (eg, $5^{\circ}-40^{\circ}$ ) as opposed to the $90^{\circ}$ flip angle used in SE. As a consequence, longitudinal magnetisation is not completely 
Figure 10 Spin echo. Following a $90^{\circ}$ excitatory radiofrequency (RF) pulse with consequent transverse magnetisation, protons begin to de-phase $(A-C)$ and signal amplitude decreases. Application of an $180^{\circ} \mathrm{RF}$ pulse at time $\mathrm{TE} / 2$ causes the protons to precess in the opposite direction. Protons that were initially 'in front' now find themselves at the back but begin to 'catch' the other protons, which are precessing at a slighter slower frequency (D-F). At time TE the protons regain phase, resulting in a stronger net transversal magnetisation and thus a stronger signal. This signal re-emergence is termed spin echo. This figure is only reproduced in colour in the online version.

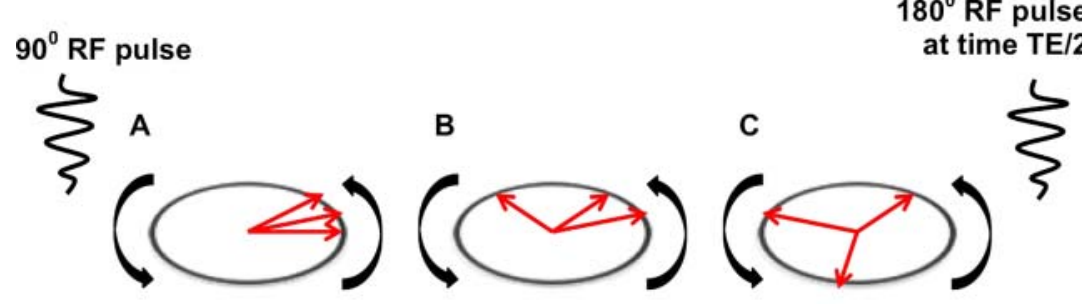

Time

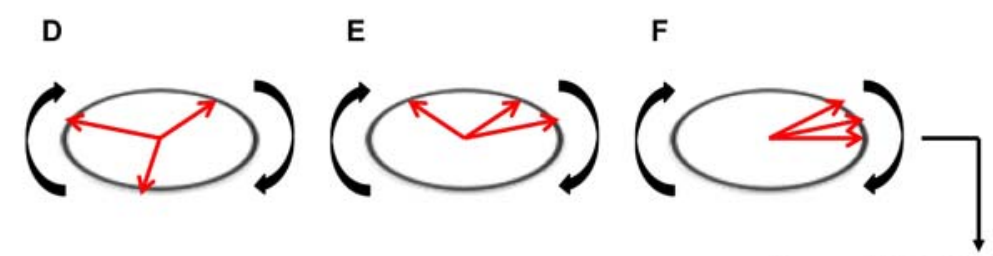

Record MR signal at time TE abolished with GRE and will provide reasonable signal even at very short TR. This combination of short TE and short TR permits rapid signal acquisition with GRE imaging. The price of short acquisition time in GRE, however, is greater signal loss in the presence of magnetic susceptibility effects. Gradient reversal refocuses only those spins that have been de-phased by the action of the gradient itself and unlike SE, where phase shifts resulting from local magnetic field inhomogeneity are minimised through the $180^{\circ} \mathrm{RF}$ pulse, this inhomogeneity persists in GRE. Hence, image contrast in GRE is influenced by T2* effects and is not confined to true T2 relaxation. ${ }^{10}$

In GRE imaging, T1- and T2*-weighted images can be obtained by manipulating the flip angle, TE and TR. This is discussed further in the section on magnetic susceptibility.

\section{IMAGE CONSTRUCTION}

\section{Localising and encoding MR signals}

The MR signal is localised in three dimensions using three separate magnetic field gradients termed (1) slice-selection gradient,

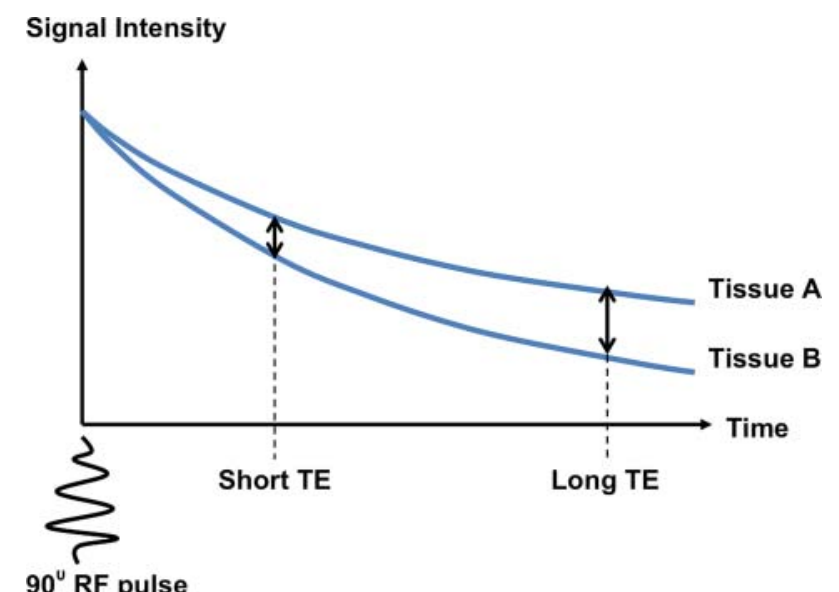

Figure 11 Echo time (TE) and T2-weighting. T2 curves for two different tissues are shown. Tissue B has a shorter $\mathrm{T} 2$ relaxation time than tissue $A$. The difference in signal intensity between the tissues is more discernible at long TE than at short TE. RF, radiofrequency. This figure is only reproduced in colour in the online version.
(2) phase-encoding gradient $\left(G_{P}\right)$ and (3) frequency-encoding gradient $\left(\mathrm{G}_{\mathrm{F}}\right)$.

Slice-selection gradient

Slice localisation is achieved by using gradient coils to generate a gradient field orientated along a chosen axis. This gradient field alters the strength of $\mathrm{B}_{0}$ in the chosen direction, so that protons within the gradient field have different Larmor frequencies (figure 14A).

Typically, the RF pulse is applied as a small range of frequencies (bandwidth) rather than as a single frequency. This excites a slice of a certain thickness. Slice thickness can be altered in two ways: (1) by changing the bandwidth of the RF pulse or (2) by changing the steepness of the gradient field.

With a given slice selectively excited, the signals arising from each slice element (pixel) within that section need to be spatially encoded. This is achieved using phase- and frequencyencoding gradients.

\section{Phase-encoding gradient}

Following an excitatory RF pulse, the protons precess in phase. Applying a new gradient magnetic field will make some of the protons precess faster than others depending on their position within the gradient. When the gradient is switched off, all protons will express the same precession frequency and will emit the same signal but they will no longer be in phase and this allows the protons to be differentiated. The gradient applied is called the $G_{P}$ and the direction of application is termed the phase-encoding direction. Thus, spin phases will vary linearly over the phase-encode direction (figure 14B).

\section{Frequency-encoding gradient}

The $G_{F}$ is applied following and perpendicular to the phase-encoding gradient. It causes the protons to rotate at different frequencies according to their relative position along the gradient, permitting differentiation of the signal in a third plane (figure 14C).

Two additional gradient pulses are typically used in this threestep process, one immediately after the slice-selection gradient and the other immediately before the $\mathrm{G}_{\mathrm{F}}$. These pulses are used to counteract any de-phasing of the transverse magnetisation 


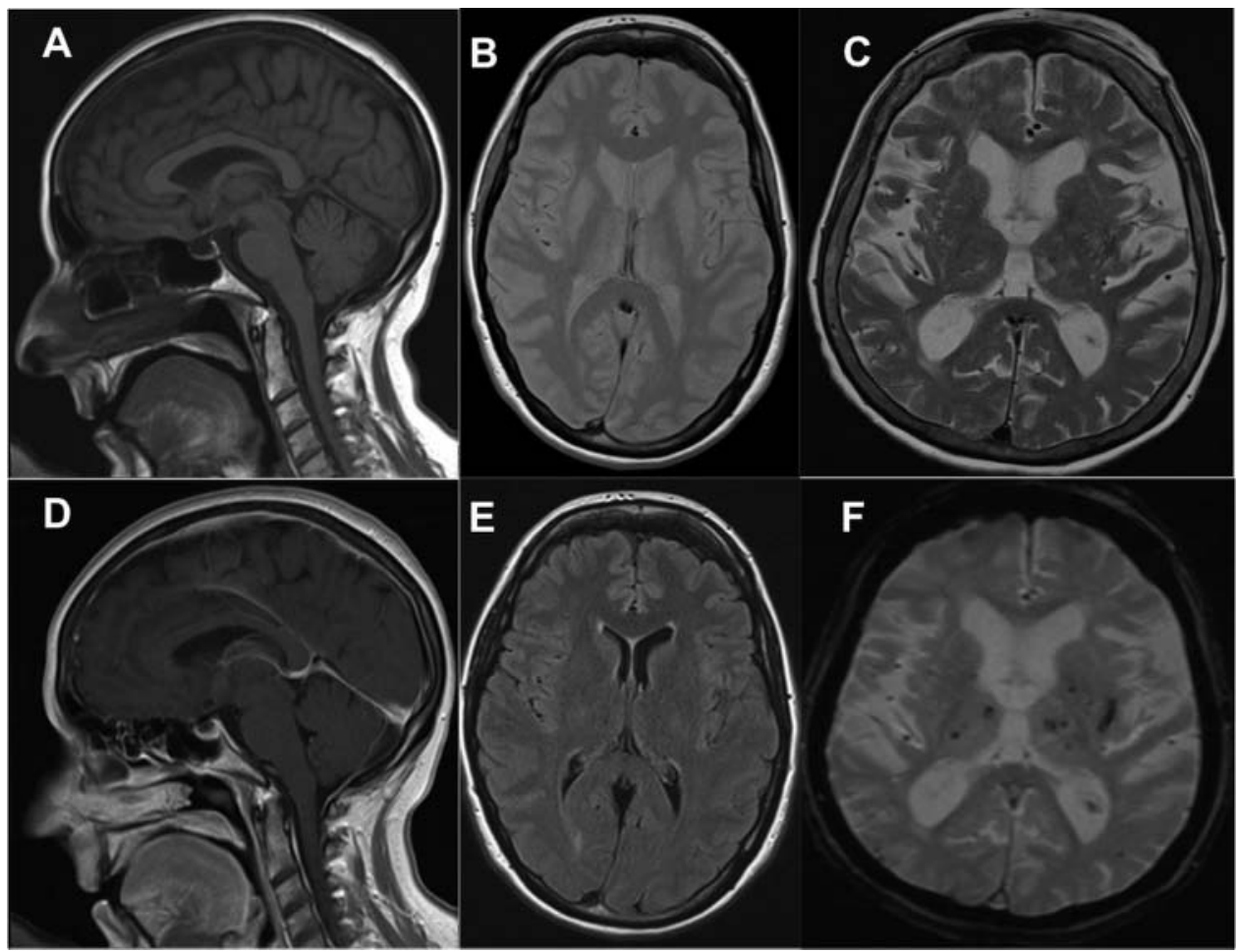

Figure 12 Examples of typical clinical MRI. (A). Sagittal T1-weighted image. Note that fat (around scalp and neck) is bright and cerebrospinal fluid (CSF) is dark. (B) Axial proton density image. Note minimal contrast between grey matter and CSF. (C) Axial T2-weighted image. Note that CSF is bright. (D) Same image as (A) but following gadolinium administration. Note the dural venous sinuses appear bright due to T1 shortening. (E) Axial fluid attenuated inversion recovery image. Note suppression of the CSF signal. (F) Axial gradient echo T2* image. Same patient as in (C). Note the increase conspicuity of multiple dark foci due to magnetic susceptibility of haemosiderin deposition in a patient with amyloid angiopathy.

that may be caused by the imaging gradients and ensures that maximum echo (sampling signal) is achieved.

It should be emphasised that RF pulses excite all protons in a slice simultaneously and that a single echo signal is recorded from the entire slice for one phase-encoding step. Thus, to acquire sufficient phase-encoding information for a signal to be assigned to each location within the slice, the pulse sequence (comprising slice selection, frequency encoding and phase encoding) is repeated many times. During each repetition, the same slice selection and frequency encoding are performed but the strength of the phase-encoding gradient is increased by equal increments. Each repetition of the phase-encoding step generates a signal echo that is digitised and stored in a raw data matrix called ' $k$-space'. Data points in k-space represent the spatial frequencies content of an MRI. Data in k-space are converted into an image using a mathematical tool called a Fourier transform.

\section{IMAGE ACQUISITION TIME}

As each phase-encoding step requires a new pulse sequence the total image acquisition time will depend on the product of TR (time interval between pulse sequences) and $\mathrm{N}_{\mathrm{P}}$ (number of phase-encoding steps). Conventional pulse sequences such as SE and GRE acquire only one phase-encoding step (one line of $\mathrm{k}$-space) per TR, making the image acquisition time considerably long. This limitation is overcome by faster imaging techniques that acquire multiple lines of k-space per TR. The so-called Turbo (or fast) SE and GRE techniques are now commonplace in MRI.

\section{Turbo SE}

The turbo SE (TSE) sequence applies multiple $180^{\circ}$ pulses after an initial $90^{\circ}$ pulse (as opposed to the single $180^{\circ}$ pulse used in a conventional SE sequence). Each $180^{\circ}$ pulse generates an echo, which (after phase encoding) is used to fill a new line of $\mathrm{k}$-space. The number of $180^{\circ}$ refocusing pulses (and, thus, the number of echoes generated) during one TR of the TSE sequence is termed the echo train length (ETL), echo factor or turbo factor. ETL specifies the factor by which the pulse sequence is accelerated (eg, an ETL of 16 means that the TSE sequence is 16 times faster than a conventional SE sequence).

The signal amplitude of each successive echo generated by the TSE sequence will be smaller than the last by way of T2 decay effects. The echoes will also have different TEs. This means that in the traditional sense, a single TE does not exist for the TSE sequence. Instead, an effective TE or pseudo TE is ascribed. The effective TE is defined as that of the echo, which is acquired closest to the centre of $\mathrm{k}$-space (with the smallest $\mathrm{G}_{\mathrm{P}}$ ) as this is the echo that has the greatest influence on image contrast. ${ }^{1}$

\section{Echo-planar imaging}

Echo-planar imaging (EPI) is a fast MRI technique in which the rapid oscillation of high-amplitude gradients is used to generate multiple GREs per TR. In single-shot EPI all of the phase-encoding steps are acquired in a single TR. Multi-shot EPI uses a few TRs to acquire all phase-encoding steps.

\section{D encoding}

Although the selection of a set of slices encodes data in all three dimensions in space, the slice thickness (often several millimetres) is often much greater than the inplane pixel resolution (often $<1 \mathrm{~mm}$ ). The initial slice-encoding step can be replaced by a further (termed secondary) phase-encoding iteration, along what was the slice-encoding direction. Instead of a set of slices 


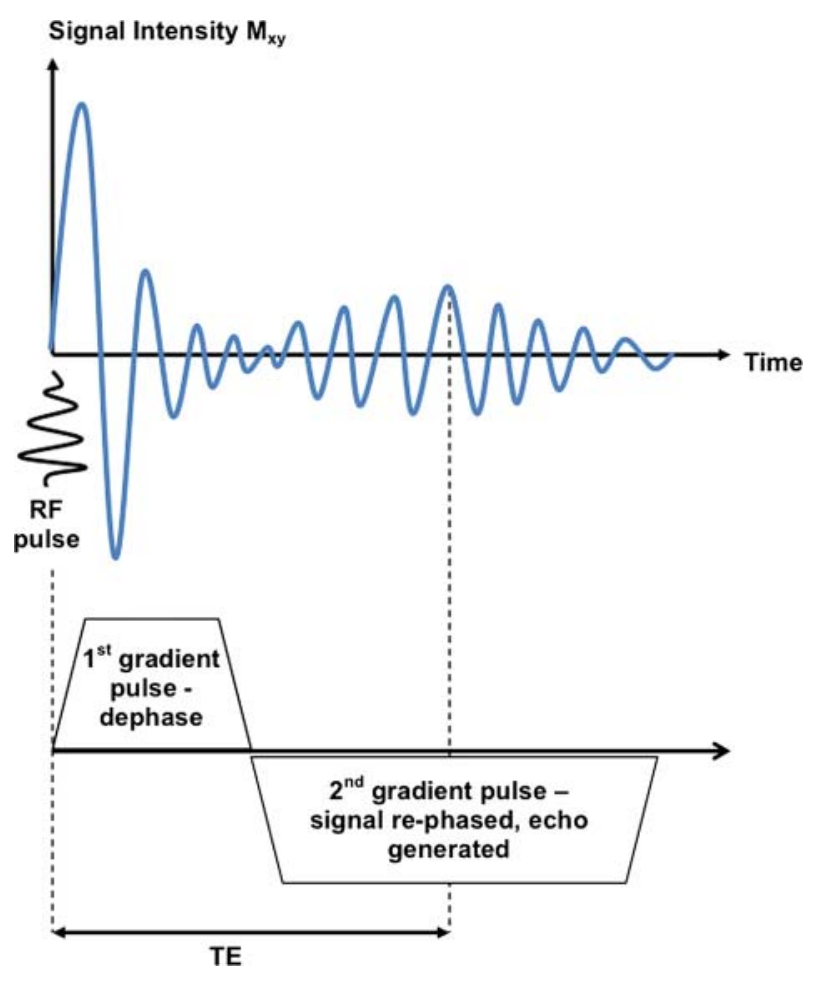

Figure 13 Gradient echo. An excitatory radiofrequency (RF) pulse causes transverse magnetisation and initiation of a free induction decay signal. This signal rapidly de-phases following the application of a magnetic field gradient. Application of a second magnetic field gradient with a slope of equal amplitude but in opposite direction to the first causes some rephasing. The signal increases again at time TE to a maximal signal termed a gradient echo. The maximum amplitude of the gradient echo is dependent on the specified TE and the $\mathrm{T} 2{ }^{*}$ relaxation rate of the tissues in question. This figure is only reproduced in colour in the online version.

(that may include slice gaps!), the inclusion of secondary phase encoding often produces a true 3D dataset comprised of voxels (3D pixels) whose dimensions can be $<1 \mathrm{~mm} \times 1 \mathrm{~mm} \times 1 \mathrm{~mm}$. This is often useful when user manipulation of data in all three perpendicular or oblique planes is necessary (eg, for intraoperative surgical guidance). Performing more phase-encoding steps has a cost: time. As a result, the majority of true $3 \mathrm{D}$ sequences tend to be GREs with short TR or TSEs with high ETL factors. ${ }^{11}$

\section{MAGNETIC SUSCEPTIBILITY}

Magnetic susceptibility describes the extent to which a substance becomes magnetised when placed in an external magnetic field. It results primarily from the interaction between electrons within the substance and the external magnetic field. More specifically, orbital and delocalised electrons within the substance produce circulating currents in response to the field. These currents generate internal magnetisation $\left(\mathrm{M}_{\mathrm{i}}\right)$ within the substance that either augments or opposes the local external magnetic field. ${ }^{4}$ The result is either positive susceptibility, whereby the magnetic field local to the substance is increased, or negative susceptibility in which the magnetic field local to the substance is decreased. Substances with negative susceptibility are termed diamagnetic; those with positive susceptibility are called paramagnetic.

Most biological tissues including water cause very weak diamagnetism and as such induce negligible susceptibility effects. Conversely, matter such as iron, nickel and cobalt show extreme positive susceptibility and are termed ferromagnetic. Superparamagnetic is used to describe a substance with positive magnetic susceptibility that lies between paramagnetism and ferromagnetism (eg, small ferrous sulphate particles).

\section{Intravenous contrast}

Gadolinium, a rare-earth metal and paramagnetic substance, is used as an intravenous MR contrast medium. Its strong paramagnetic properties result from the numerous unpaired electrons that exist within its inner shells. These unpaired electrons can interact with adjacent resonating protons and cause the protons to relax more rapidly. This results in a shortening of both longitudinal and transverse relaxation with consequent reduction in the $\mathrm{T} 1$ and $\mathrm{T} 2$ values of the tissues in which it accumulates. This is depicted by an increase in signal in T1-weighted images and a decrease in signal in T2-weighted images. In practice, the signal increase detected on T1-weighted imaging is better appreciated than any corresponding signal decrease in T2-weighted imaging and makes T1-weighted imaging the method of choice for intravenous contrast studies. Furthermore, as gadolinium-based contrast agents shorten T1, it is possible to also shorten TR and, hence, reduce overall scan time (figure 12D).

\section{Exploiting magnetic susceptibility using GRE imaging}

Susceptibility differences among tissues and materials are more conspicuous on T2*-weighted GRE rather than SE imaging (recall that susceptibility differences promote magnetic field inhomogeneity that result in faster $\mathrm{T} 2 *$ relaxation, leading to signal intensity loss on GRE images; conversely, the $180^{\circ}$ refocusing pulse in SE compensates for this). GRE sequences can be made more sensitive to $\mathrm{T} 2 *$ decay through the manipulation of imaging parameters such as TR, TE and the flip angle. As $\mathrm{T} 2 *$ decay commences with the excitation pulse and progresses with time, a longer TE will result in greater signal loss. A low flip angle reduces the influence T1 has on the subject matter (as longitudinal magnetisation remains close to the fully relaxed state) and allows the T2* effects to dominate. Similarly, T1 effects are limited with a long TR. Hence, T2* GRE imaging is obtained at long TE, long TR and low flip angle. T2* sensitivity also increases with field strength and voxel size. ${ }^{12}$ T2*-weighted sequences are particularly useful in the detection of haemorrhage and blood products (deoxyhaemoglobin, methaemoglobin and haemosiderin are paramagnetic). Various pathologies may be detected using this imaging modality including arteriovenous malformations, cavernomas, superficial siderosis, intraparenchymal/intratumoural haemorrhage and amyloid angiopathy (figure 12F). The literature also supports the use of T2*-weighted imaging for the differentiation of vestibular schwannomas from meningiomas as in most schwannomas, microhaemorrhages can be detected. ${ }^{13}$

\section{OTHER FACTORS THAT MAY INFLUENCE SIGNAL INTENSITY}

\section{TI: the inversion recovery sequence}

The inversion recovery sequence uses a $180^{\circ}$ pulse followed by a $90^{\circ}$ pulse. The $180^{\circ}$ pulse inverts longitudinal magnetisation so that the sum magnetisation aligns antiparallel to $B_{0}$. Following the $180^{\circ}$ pulse, the inverted longitudinal magnetisation begins to shorten and return to its original position. Application of a $90^{\circ}$ pulse causes the longitudinal magnetisation to flip to the transverse plane and allows a signal to be recorded. The strength of the signal is dependent on the magnitude of longitudinal magnetisation just before the $90^{\circ}$ pulse was applied. 


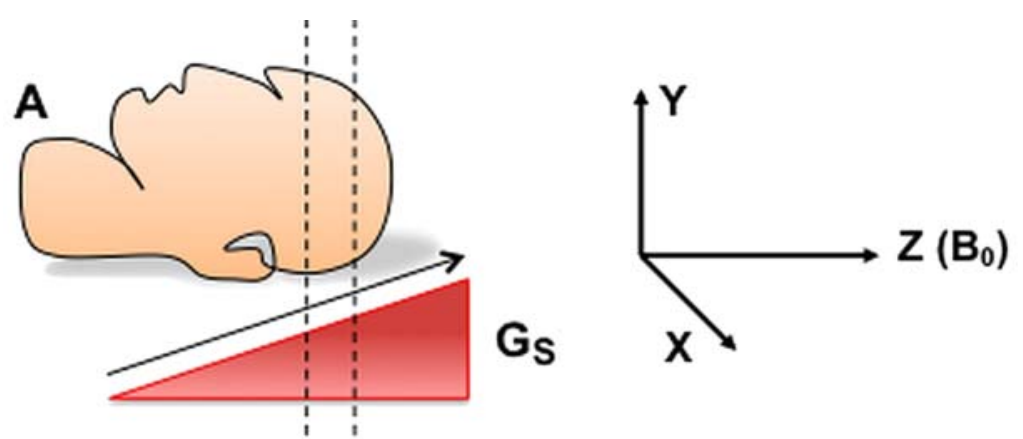

Figure 14 Image construction. (A) Step 1: Slice selection. A slice-selecting gradient $\left(G_{s}\right)$ is applied at the same time as the excitatory radiofrequency (RF) pulse. In this example, the brain is being imaged and $G_{S}$ is applied along the $z$-axis, parallel with $B_{0}$. This means that different cross sections of the brain experience magnetic fields of differing strength. Accordingly protons will precess at different Larmor frequencies depending on their position along the gradient. Selecting an RF pulse (or range of RF pulse frequencies) that matches the Larmor frequency of the protons will determine the slice location. (B) Step 2: Phase encoding. Protons are in phase after the RF pulse is applied. Applying a phase-encoding gradient along the $y$-axis causes the protons to increase their speed of precession relative to the strength of the magnetic field to which they are exposed. In this example, speed increases from top to bottom. This is depicted on the right of the schematic, which shows rows of protons with different precession speeds. When the gradient is switched off, all protons are once again exposed to the same magnetic field and as such they have the same frequency; only now, they are out of phase. (It may help to think of the protons giving of the same signal frequency but at different times.) (C) Step 3: Frequency encoding. A further magnetic field frequency-encoding gradient is applied to help differentiate the signal from different protons by way of differing frequencies. In this example, the protons from the bottom row in step 2 (above) have been exposed to a gradient applied along the $x$-axis. While the protons are in phase, they now have different frequencies, allowing their differentiation in the third ( $\mathrm{x}$ ) plane. During the frequency-encoding step, the signal is measured (digitally sampled) at time TE, the point of maximal signal. The signal comprises a range of frequencies (or bandwidth) that correspond to the Larmor frequencies of the proton magnetic moments at their different locations along the gradient. ${ }^{1}$ In this example, the orientation of the three gradients defined a slice perpendicular to the z-axis. Different slice orientations can be achieved by allocating the gradients to a different axis. Combining gradients along two or more axes for each localisation task allows angled slices to be obtained. This figure is only reproduced in colour in the online version. 
Hence, the signal obtained by inversion recovery is dependent on the T1 properties of the tissues being examined. Inversion time (TI) is the time interval between the $180^{\circ}$ and the $90^{\circ}$ pulses (figure 15A).

Following the $180^{\circ}$ pulse there will be a time when longitudinal magnetisation passes through the transverse plane. Application of a $90^{\circ}$ pulse at this point will result in a signal void (the protons will be flipped out of the orientation at which their signal can be recorded). Accordingly, this point is termed the null point. Knowledge of the null point for a particular tissue can be used to suppress the signal from that tissue (figure 15B). Fluid attenuated inversion recovery (FLAIR) sequences are frequently used in neuroimaging to suppress the signal from cerebrospinal fluid (figure 12D). This has the particular advantage of making periventricular lesions much more conspicuous. As a further bonus, the combination of long TE and a TI can provide both T2-weighted contrast (aiding the delineation of, for example, ischaemia or inflammation) and T1-weighted contrast (showing gadolinium-chelate deposition where there is a leaky blood-brain barrier). Gadolinium enhanced FLAIR sequences are being used with increasing frequency in neuroscience centres. They can be particularly useful in the detection of subtle tumours or for assessing multiple sclerosis. Short TI inversion recovery is used commonly throughout radiology subspecialties to suppress fat signal. Other fat-suppression techniques are available. ${ }^{14}$

\section{Flow}

When imaging a slice through a vessel containing flowing blood, the blood within the slice at the time of an excitatory RF pulse will be influenced by the radiowave. However, by the time the
MR signal is sampled, the blood influenced by the radiowave will have been replaced by blood flowing into the slice that has not undergone magnetisation. Thus, no signal will be obtained from the vessel and it will appear black on the image: the so-called flow-void phenomenon.

This is not the only way in which flow influences signal intensity and merely constitutes an introduction as in reality the subject is far more complex. For example, a signal may still be obtained from vessels that contain slow flowing blood and this to the inexperienced looking at a single sequence of images may be interpreted as thrombosis. Detailed articles describing MRI in relation to flow and articles relating to MRI artefacts can be found in the literature. ${ }^{15} 16$

\section{FINALLY, A WORD ON SAFETY}

Safety issues regarding clinical MRI can be broadly divided into those that result from the exposure to magnetic fields and those resulting from intravenous contrast administration.

\section{Exposure to magnetic fields}

Each of the three types of magnetic field (static, gradient and $\mathrm{RF}$ ) that the patient is exposed to during an examination carries a potential risk to patient safety. With regard to the static magnetic field $\left(\mathrm{B}_{0}\right)$, the main safety issue involves the attraction of ferromagnetic material towards the magnet. Material external to the patient (such as keys or coins) may become projectiles within the scanning room, putting the patient and staff (and machinery) at risk of being hit. Static magnetic fields can also exert mechanical forces on ferromagnetic components within implanted medical devices such as pacemakers, aneurysmal clips and cardiac defibrillators. Devices may rotate or dislodge,
Figure 15 Inversion recovery. (A) Inversion of the sum longitudinal magnetisation vector following an $180^{\circ}$ radiofrequency (RF) pulse. A further $90^{\circ} \mathrm{RF}$ pulse flips the recovering sum vector into the transverse plane allowing a signal to be obtained. The magnitude of the signal is dependent on the amount of $\mathrm{T} 1$ recovery that had occurred before the $90^{\circ}$ pulse and, hence, on the inversion time (time between the $180^{\circ}$ and $90^{\circ}$ pulses). (B) T1 relaxation curves for fat and water demonstrating the recovery of longitudinal magnetisation following an $180^{\circ} \mathrm{RF}$ pulse. Application of a $90^{\circ} \mathrm{RF}$ pulse at the null point leads to signal suppression of a chosen tissue. This figure is only reproduced in colour in the online version.
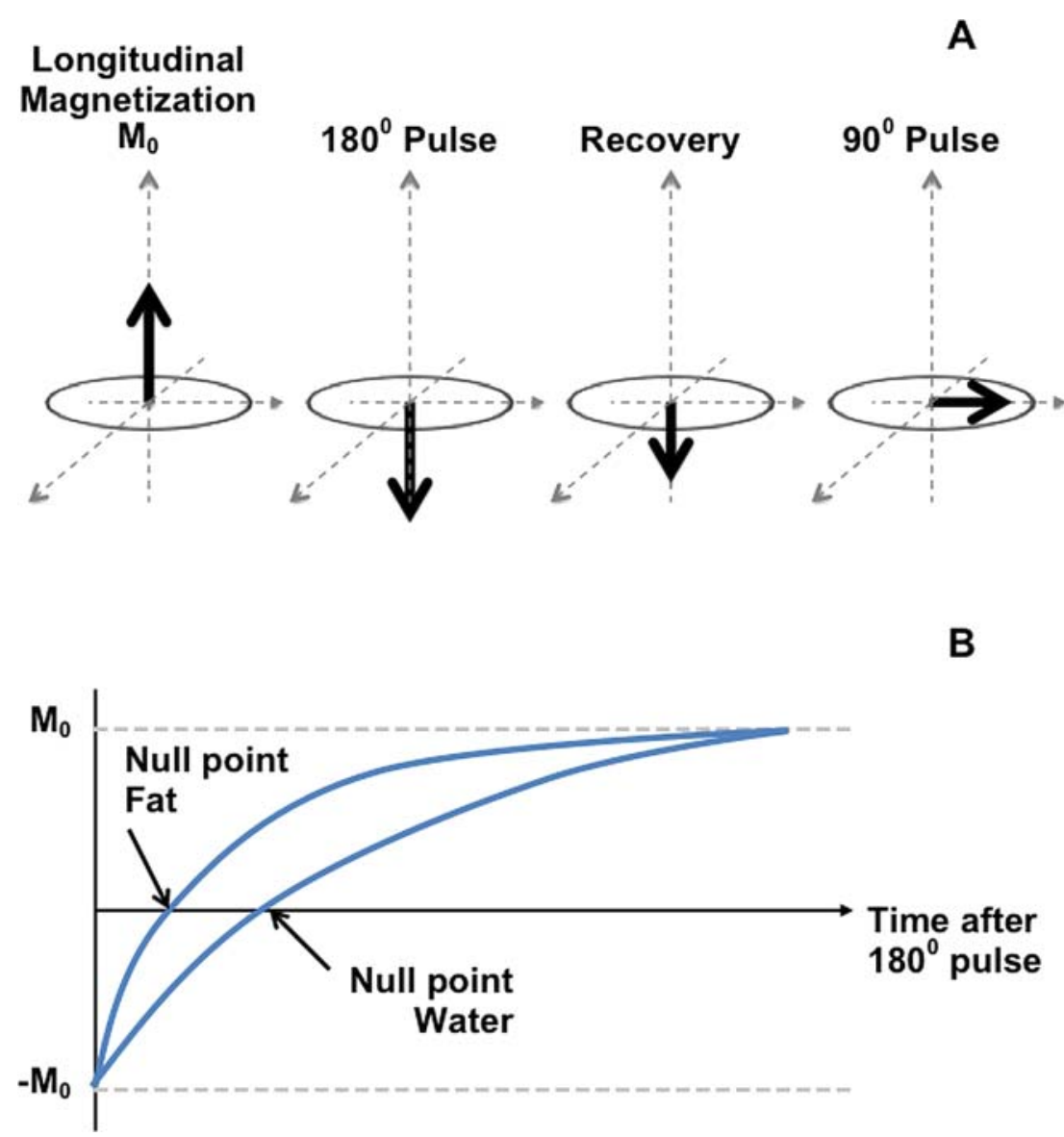
potentially resulting in serious patient harm. ${ }^{17}$ Higher static magnetic fields lead to greater forces on ferromagnetic materials. ${ }^{18}$ Control of access to the magnet room is crucial in minimising associated risks.

Excessive noise and potential auditory damage are the main concerns with gradient fields. Rapid alterations of currents within the gradient coils, in the presence of a strong magnetic field, generate significant force. This force acts upon the gradient coils and manifests as loud tapping or knocking sounds. ${ }^{19}$ Temporary hearing loss has been reported using conventional sequences. ${ }^{20}$ Due to the risk of damage caused by acoustic noise it is common practice for the patient to wear protective ear wear (often headphones and earplugs). Gradient magnetic fields also have the potential to induce voltages on pacing wires that may cause oversensing and undersensing. Excitation of peripheral nerves has also been reported. ${ }^{21}$

RF pulses may lead to local tissue heating through the dissipation of energy. Typically this is negligible $\left(<1^{\circ} \mathrm{C}\right)$ in clinical imaging but is potentially serious in patients with implantable devices. A pacemaker lead, for example, may act as an antenna and concentrate RF energy, resulting in dramatic local heating, tissue damage and cardiac dysfunction. ${ }^{22}$

A devise must have undergone prior testing within MR electromagnetic fields for it to be deemed safe. Booklets listing MR compatible objects/devises are available and are typically held in the MR scanner room. More and more MR compatible medical devices and equipment are being generated. Most tertiary centres now have MR-safe anaesthetic equipment and recent studies have reported favourably on the early outcome of MR compatible pacemakers. ${ }^{17}$

\section{Intravenous contrast agents}

Gadolinium-based contrast agents are generally well tolerated. A recent study suggests an adverse reaction rate of $0.46 \% .{ }^{23} \mathrm{~A}$ rare but serious complication that may occur in patients with severe renal impairment is nephrogenic systemic fibrosis. ${ }^{24}$ Although a systemic condition, cutaneous manifestations tend to dominate with a lower-limb cellulitic-like picture occurring early. Painful and pruritic, plaque-like 'woody' induration of the skin occurs later in the course of the disease with the legs more commonly affected than the arms and torso. The face is spared. ${ }^{25}$ These cutaneous manifestations may lead to contractures and limit mobility. nephrogenic systemic fibrosis may also affect the liver, lungs, muscles, heart and nerves. ${ }^{26}$ Death rate may be as high as $28 \% .^{27}$ Despite ongoing research the exact mechanisms underlying the condition remain unclear. No universally effective treatment has been identified and prevention (mainly through limiting the use of high-dose gadolinium-based contrast agents and/or maximising renal function in patients with renal failure) represents current recommendations. ${ }^{25}$

Anaphylaxis resulting from gadolinium-based contrast agents is rare, with an estimated incidence of $0.02 \% .^{23} 2829$ This compares favourably to the incidence with iodinated contrast media used with CT $(0.03 \%-0.16 \%) .{ }^{29}$ Patients with a pre-existing allergy to other contrast agents (such as iodine) may confer a greater risk of anaphylaxis to gadolium-based contrast agents, thus highlighting the need for a thorough screening questionnaire prior to any contrast administration. ${ }^{23} 25$

\section{Pregnancy and breast feeding}

Large doses of gadolinium-based contrast agents have been shown to cause postimplantation fetal loss, retarded development, increased locomotive activity and skeletal and visceral abnormalities in animal experiments. ${ }^{19}$ Any deleterious effects to humans are unconfirmed. However, gadolinium-based contrast agents can cross the placenta and appear in the fetal bladder from where they are excreted but enter the fetal system again through the swallowing of amniotic fluid. This makes it difficult to ascertain exact fetal half-life measurements for these agents. ${ }^{30}$ Currently, it is recommended that gadolinium-based contrast should only be administered after careful risk-benefit assessment. ${ }^{25}$

The amount of contrast absorbed by an infant's gastrointestinal tract following breast feeding is believed to be negligible $(<1 \%$ of the recommended intravenous infant dose) and as such no additional precautions are thought necessary for breastfeeding mothers. ${ }^{31}$ Some centres advise informed consent, however, with an option to stop breast feeding (with continued expression and discarding of the milk) for $24 \mathrm{~h}$ following maternal administration. ${ }^{31}$

\section{SUMMARY}

This article has outlined the fundamental physical principles involved in clinical MRI. Key imaging parameters and sequences have been described, including their influence on image contrast and acquisition time. Important safety issues have also been addressed.

\section{Main messages}

- More frequently hospital clinicians are reviewing images from MR studies of their patients before seeking formal radiological opinion.

- Knowledge of the basic physical principles behind MRI is essential for correct image interpretation.

- The use of MRI as an investigative tool will only increase in clinical medicine.

\section{Current research questions}

- Can advanced MR imaging techniques fulfill their potential as specific and sensitive biomarkers of disease?

- Will new image reconstruction algorithms (that move on from the simple Fourier transform) generate improved, artifact-free images that can be applied in clinical radiology?

\section{Key references}

- Elster A. Questions and answers in magnetic resonance imaging. St Louis: Mosby-Year Book, 1994.

- Chavhan GB, Babyn PS, Thomas B, et al. Principles, techniques, and applications of T2*-based MR imaging and its special applications. Radiographics 2009;29:1433-49.

- Bley TA, Wieben 0, Francois CJ, et al. Fat and water magnetic resonance imaging. J Magn Reson Imaging 2010;31:4-18.

- Ivancevic MK, Geerts L, Weadock WJ, et al. Technical principles of MR angiography methods. Magn Reson Imaging Clin N Am 2009;17:1-11.

- Coskun 0. Magnetic resonance imaging and safety aspects. Toxicol Ind Health 2011;27:307-13. 


\section{MULTIPLE CHOICE QUESTIONS (TRUE (T)/FALSE (F); ANSWERS AFTER THE REFERENCES)}

1.

A. The room housing the MR machine is typically lined with copper?

B. Clinical MR systems typically use superconducting magnets?

C. The main magnetic coils generate a strong constant magnetic field referred to as $\mathrm{B}_{1}$ ?

D. Gradient coils are cooled, close to absolute zero, using cryogenic liquid helium?

E. Shim coils are used to reduce inhomogeneity?

2.

A. When placed in an external magnetic field protons move in a particular way called precession?

B. The speed of precession is determined by the Larmor equation?

C. The Larmor equation indicates that precession frequency is proportional to magnetic field strength?

D. Longitudinal magnetisation refers to a sum magnetisation that parallels the external magnetic field?

E. Longitudinal magnetisation can be directly measured to create an MR signal?

3.

A. Activation of a $90^{\circ}$ radiofrequency pulse flips the sum magnetisation vector to the right creating transverse magnetisation?

B. T1 relaxation is the process whereby protons exchange energy with their surroundings to return to their lower energy state and in so doing cause the restoration of transverse magnetisation?

C. T2 relaxation results from inhomogeneity within the local tissue?

D. T2* relaxation determines the actual rate of decay observed when measuring a free induction decay signal?

E. T2 is a constant describing the time taken for transverse magnetisation to decay to about $63 \%$ of its initial value?

4.

A. Short TR and short TE generate a predominantly T1-weighted image?

B. Long TR and long TE produce a predominantly T2-weighted image?

C. Short TR and long TE are used to generate a proton density image?

D. Spin echo sequences generally have greater magnetic susceptibility effects than gradient echo sequences?

E. Gradient echo sequences use a $90^{\circ}$ saturation pulse followed by a $180^{\circ}$ refocusing pulse?

5.

A. Substances with negative magnetic susceptibility are termed paramagnetic?

B. Iron is termed ferromagnetic because of its extreme negative magnetic susceptibility?

C. The signal obtained by inversion recovery is dependent on the T1 properties of the tissues being examined?

D. Excessive noise and potential auditory damage are the main concerns with radiofrequency coils?

E. Gadolinium-based contrast agents have an adverse reaction rate of about $0.5 \%$ ?

Contributors SC: concept design, literature review, manuscript drafting, manuscript revision. NH and IDW: concept design, manuscript revision. IJC and MH: manuscript revision.
Funding None.

Competing interests None.

Provenance and peer review Not commissioned; externally peer reviewed.

\section{REFERENCES}

1 Ridgway JP. Cardiovascular magnetic resonance physics for clinicians: part I. J Cardiovasc Magn Reson 2010;12:71.

2 Jacobs MA, Ibrahim TS, Ouwerkerk R. AAPM/RSNA physics tutorials for residents: MR imaging: brief overview and emerging applications. Radiographics 2007:27:1213-29.

3 Hahn EL. Spin Echoes. Phys Rev 1950;80:580-94.

4 Elster A. Questions and answers in magnetic resonance imaging. St Louis: Mosby-Year Book, 1994.

5 Schild H. MRI made easy. Berlin: Schering, 1990

6 Smith H, Ranello F. A non-mathematical approach to basic MRI. Madison, Wis: Medical Physics Publishing, 1989.

7 Bloch F. Nuclear induction. Phys Rev 1946;70:460.

8 Fullerton G. Physiologic basis of magnetic relaxation. In: Stark D, Bradley W, eds. Magnetic resonance imaging. 2nd edn. St Louis: Mosby-Year Book, 1992: 88-108.

9 Pikus L, Woo JH, Wolf RL, et al. Artificial multiple sclerosis lesions on simulated FLAIR brain MR images: echo time and observer performance in detection. Radiology 2006;239:238-45.

10 Winkler ML, Ortendahl DA, Mills TC, et al. Characteristics of partial flip angle and gradient reversal MR imaging. Radiology 1988;166(1 Pt 1):17-26.

11 Mugler JP III, Brookeman JR. Three-dimensional magnetization-prepared rapid gradient-echo imaging (3D MP RAGE). Magn Reson Med 1990;15:152-7.

12 Chavhan GB, Babyn PS, Thomas B, et al. Principles, techniques, and applications of T2*-based MR imaging and its special applications. Radiographics 2009:29:1433-49.

13 Thamburaj K, Radhakrishnan VV, Thomas B, et al. Intratumoral microhemorrhages on $2^{*}$-weighted gradient-echo imaging helps differentiate vestibular schwannoma from meningioma. AJNR Am J Neuroradiol 2008;29:552-7.

14 Bley TA, Wieben 0 , Francois CJ, et al. Fat and water magnetic resonance imaging J Magn Reson Imaging 2010;31:4-18.

15 Ivancevic MK, Geerts L, Weadock WJ, et al. Technical principles of MR angiography methods. Magn Reson Imaging Clin N Am 2009;17:1-11.

16 Morelli JN, Runge VM, Ai F, et al. An image-based approach to understanding the physics of MR artifacts. Radiographics 2011;31:849-66.

17 Jung W, Zvereva V, Hajredini B, et al. Initial experience with magnetic resonance imaging-safe pacemakers : a review. J Interv Card Electrophysiol 2011;32:213-19.

18 Pamboucas C, Nihoyannopoulos P. Cardiovascular magnetic resonance at 3 Tesla: advantages, limitations and clinical potential. Hellenic J Cardiol 2006;47:170-73.

19 Coskun 0 . Magnetic resonance imaging and safety aspects. Toxicol Ind Health 2011;27:307-13.

20 Chakeres DW, de Vocht F. Static magnetic field effects on human subjects related to magnetic resonance imaging systems. Prog Biophys Mol Biol 2005;87:255-65.

21 Pamboucas CA, Rokas SG. Clinical safety of cardiovascular magnetic resonance: cardiovascular devices and contrast agents. Hellenic J Cardiol 2008;49:352-6.

22 Baikoussis NG, Apostolakis E, Papakonstantinou NA, et al. Safety of magnetic resonance imaging in patients with implanted cardiac prostheses and metallic cardiovascular electronic devices. Ann Thorac Surg 2011;91:2006-11.

23 Li A, Wong CS, Wong MK, et al. Acute adverse reactions to magnetic resonance contrast media-gadolinium chelates. Br J Radiol 2006;79:368-71.

24 Cowper SE, Robin HS, Steinberg SM, et al. Scleromyxoedema-like cutaneous diseases in renal-dialysis patients. Lancet 2000;356:1000-1.

25 Gauden AJ, Phal PM, Drummond KJ. MRI safety: nephrogenic systemic fibrosis and other risks. J Clin Neurosci 2010;17:1097-104.

26 Gibson SE, Farver CF, Prayson RA. Multiorgan involvement in nephrogenic fibrosing dermopathy: an autopsy case and review of the literature. Arch Pathol Lab Med 2006;130:209-12.

27 Mendoza FA, Artlett CM, Sandorfi N, et al. Description of 12 cases of nephrogenic fibrosing dermopathy and review of the literature. Semin Arthritis Rheum 2006:35:238-49.

28 Murphy KJ, Brunberg JA, Cohan RH. Adverse reactions to gadolinium contrast media: a review of 36 cases. AJR Am J Roentgenol 1996;167:847-9.

29 Caro JJ, Trindade E, McGregor M. The cost-effectiveness of replacing high-osmolality with low-osmolality contrast media. AJR Am J Roentgenol 1992;159:869-74.

30 Shellock F, Kanal E. Bioeffects and safety of MR procedures. In: Edelman R, Hesselink J, Zlatkin M, eds. Clinical magnetic resonance imaging. Philadelphia: W.B. Saunders, 1996: 429.

31 Webb JA, Thomsen HS, Morcos SK. The use of iodinated and gadolinium contrast media during pregnancy and lactation. Eur Radiol 2005;15:1234-40. 


\section{ANSWERS}

1.

A. $T$

B. $\mathrm{T}$

C. $\mathrm{F}-\mathrm{B}_{0}$

D. F - main magnet

E. $T$

2.

A. $T$

B. $T$

C. $\mathrm{T}$

D. $T$

E. F - need transverse magnetisation

3.

A. $T$

B. F - longitudinal magnetisation
C. T
D. $T$
E. $F-37 \%$
4.
A. $T$
B. $T$
C. F - long TR, short TE
D. $\mathrm{F}$ - fewer
E. $F$ - this is $S E$
5.
A. F - diamagnetic
B. F - because of its extreme positive magnetic susceptibility
C. T - but also provides T2 contrast because of long TE
D. F - gradient coils
E. T 